\title{
Monte Carlo simulations of the halo white dwarf population
}

\author{
E. García-Berro ${ }^{1,2}, \mathrm{~S}$. Torres ${ }^{1}$, J. Isern $^{2,3}$, and A. Burkert ${ }^{4}$ \\ ${ }^{1}$ Departament de Física Aplicada, Escola Politécnica Superior de Castelldefels, Universitat Politècnica de Catalunya, \\ Avda. del Canal Olímpic s/n, 08860 Castelldefels, Spain \\ 2 Institute for Space Studies of Catalonia, c/Gran Capità 2-4, Edif. Nexus 104, 08034 Barcelona, Spain \\ ${ }^{3}$ Institut de Ciències de l'Espai, CSIC \\ 4 Max-Planck-Institut für Astronomie, Koenigstuhl 17, 69117 Heidelberg, Germany
}

Received 20 October 2003 / Accepted 24 December 2003

\begin{abstract}
The interpretation of microlensing results towards the Large Magellanic Cloud (LMC) still remains controversial. While white dwarfs have been proposed to explain these results and, hence, to contribute significantly to the mass budget of our Galaxy, there are also several constraints on the role played by white dwarfs. In this paper we analyze self-consistently and simultaneously four different results, namely, the local halo white dwarf luminosity function, the microlensing results reported by the MACHO team towards the LMC, the results of Hubble Deep Field (HDF) and the results of the EROS experiment, for several initial mass functions and halo ages. We find that the proposed log-normal initial mass functions do not contribute to solve the problem posed by the observed microlensing events and, moreover, they overproduce white dwarfs when compared to the results of the HDF and of the EROS survey. We also find that the contribution of hydrogen-rich white dwarfs to the dynamical mass of the halo of the Galaxy cannot be more than $\sim 4 \%$.
\end{abstract}

Key words. stars: white dwarfs - stars: luminosity function, mass function - Galaxy: stellar content - Galaxy: dark matter Galaxy: structure - Galaxy: halo

\section{Introduction}

White dwarfs are the most common remnants of stellar evolution. Since white dwarfs are long-lived objects and the physical processes governing their evolution are relatively well understood - at least up to moderately low luminosities - they provide us with an invaluable tool to study the evolution and structure of our Galaxy. In fact, the disk white dwarf luminosity function has become an important tool to determine some properties of the local neighborhood, such as its age (Winget et al. 1987; García-Berro et al. 1988; Hernanz et al. 1994), or the past history of the star formation rate (Noh \& Scalo 1990; Díaz-Pinto et al. 1994; Isern et al. 1995a,b). This has been possible because now we have improved observational luminosity functions (Liebert et al. 1988; Oswalt et al. 1996; Leggett et al. 1998) and because we have reliable cooling sequences - see, for instance, Salaris et al. (2000), and references therein.

Although the situation for the disk white dwarf population seems to be clear and well understood, this is not the case for the halo white dwarf population. The discovery of microlenses towards the Large Magellanic Cloud (Alcock et al. 1996, 2000; Lasserre et al. 2001) has generated a large controversy about the possibility that white dwarfs could be

Send offprint requests to: E. García-Berro, e-mail: garcia@fa.upc.es responsible for these microlensing events and, thus, could provide a significant contribution to the mass budget of our Galactic halo. Nevertheless, white dwarfs as dark matter candidates are not free of problems, since an excess of this kind of object would necessarily imply an overproduction of low-mass main sequence red dwarfs and high-mass stars that could eventually explode as type II supernovae. To solve these problems Adams \& Laughlin (1996) and Chabrier et al. (1996) proposed non-standard initial mass functions in which the formation of both low and high mass stars was suppressed. Besides the lack of evidence favoring such ad-hoc initial mass functions, they are not free of problems either. In particular, the formation of a typical $\left(M \sim 0.6 M_{\odot}\right)$ white dwarf is accompanied by the injection into the interstellar medium of a sizeable amount of mass $\left(\sim 1.5 M_{\odot}\right.$ on average). Since type II supernovae are suppressed in ad-hoc initial mass functions, there is not enough energy to eject this matter into the intergalactic medium and the ejected mass, which is significantly contaminated by metals (Abia et al. 2001; Gibson \& Mould 1997), cannot be accomodated in the Galaxy (Isern et al. 1998). Finally, an excess of white dwarfs also translates into an excess of binaries containing such stars with the subsequent increase of type Ia supernova rates which, ultimately, results in an increase in the abundances of the elements of the iron peak (Canal et al. 1997). All these arguments have forced the search for other possible explanations, such as 
self-lensing in the LMC (Wu 1994; Salati et al. 1999), or background objects (Green \& Jedamzik 2002) which have not been totally discarded yet.

The suggestion of the MACHO team (Alcock et al. 1997, 2000) that white dwarfs contribute significantly to the mass budget of the Galactic halo has motivated a large number of observational searches (Knox et al. 1999; Ibata et al. 1999; Oppenheimer et al. 2001; Majewski \& Siegel 2002; Nelson et al. 2002) for these elusive white dwarfs. Also several theoretical works (Reylé et al. 2001; Flynn et al. 2003) have analyzed this possibility. However, the controversy of whether or not white dwarfs can provide a significant contribution to the Galactic dark matter is still open and deserves some more attention. In Isern et al. (1998) we analyzed the halo white dwarf population. In particular, we computed, assuming a standard initial mass function and updated models of white dwarf cooling, the expected luminosity function, both in luminosity and in visual magnitude, for different star formation rates. We showed that a deep enough survey (limiting magnitude $\gtrsim 20$ ) could provide important information about the halo age and the duration of the formation stage. We also showed that the number of white dwarfs produced using the proposed biased IMFs could not represent a large fraction of the halo dark matter if they were constrained by the observed luminosity function of halo white dwarfs. However, within the approach adopted there the biases introduced by the sample selection procedures were not taken into account. More recently, we have analyzed (Torres et al. 2002) the sample of Oppenheimer et al. (2001). In this paper we examine in detail the results of the MACHO team (Alcock et al. 2000) carefully taking into account the observational biases, thus updating our previous papers on this subject. We also study the number of white dwarfs that could be potentially found in the HDF (Ibata et al. 1999). Finally we also analyze the very recent observational results of the EROS team (Goldman et al. 2002), which set a very stringent upper limit to the white dwarf content of the Galactic halo. All these analyses are done by making use of a Monte Carlo simulator (García-Berro et al. 1999; Torres et al. 1998). This is an important issue since white dwarf populations are usually drawn from kinematically selected samples (white dwarfs with relatively high proper motions). Therefore, some kinematical biases or distortions are expected. A Monte Carlo simulation of a model population of white dwarfs is expected to allow the biases and effects of sample selection to be taken into account, so the properties of the real sample could be corrected - or, at least, correctly interpreted - provided that a detailed simulation from the very early stages of source selection is performed accurately. Our paper is organized as follows. In section Sect. 2 we describe in full detail the Monte Carlo code. In Sect. 3 we present the results, whereas in Sect. 4 our conclusions are summarized.

\section{The model}

In this section we discuss the main ingredients of our Monte Carlo simulator. Since we want to self-consistently simulate simultaneously four different results, namely, the local halo white dwarf luminosity function, the microlensing results towards the LMC, the results of the HDF, and the results of the EROS team, and each one of these simulations requires slightly different inputs, we will describe them in separate subsections. All of them, however, share some common ingredients like a random number generator, which is always at the heart of any Monte Carlo simulation. We have used a random number generator algorithm (James 1990) which provides a uniform probability density within the interval $(0,1)$ and ensures a repetition period of $\gtrsim 10^{18}$, which is enough for our purposes. Each one of the Monte Carlo simulations discussed in Sect. 3 below consists of an ensemble of 40 independent realizations of the synthetic white dwarf population, for which the average of any observational quantity along with its corresponding standard deviation were computed. Here the standard deviation means the ensemble mean of the sample dispersions for a typical sample.

\subsection{The local halo white dwarf luminosity function}

We have considered a typical spherically symmetric halo. One of the most commonly used models of this type is the isothermal sphere. The density profile of the luminous halo is given by the law

$\rho(r)=\rho_{0} \frac{a^{2}+R_{\odot}^{2}}{a^{2}+r^{2}}$

where $a \approx 5 \mathrm{kpc}$ is the core radius, $\rho_{0}$ is the local halo density and $R_{\odot}=8.5 \mathrm{kpc}$ is the galactocentric distance of the Sun. We randomly choose three numbers for the spherical coordinates $(r, \theta, \phi)$ of each star of the sample within approximately $350 \mathrm{pc}$ from the sun, according to Eq. (1). Afterwards we draw another pseudo-random number in order to obtain the main sequence mass of each star, according to one of the four model initial mass functions that will be studied here. These initial mass functions are, respectively, a standard IMF (Scalo 1998), the biased IMF of Adams \& Laughlin (1996) and the two adhoc IMFs proposed by Chabrier et al. (1996). All these mass functions are summarized in Table 1 . Once the mass of the progenitor of the white dwarf is known we randomly choose the time at which each star was born $\left(t_{\mathrm{b}}\right)$. For this purpose we assume that the halo was formed $14 \mathrm{Gyr}$ ago (see, however, Sect. 3 below) in an intense burst of star formation of duration $\sim 1$ Gyr. Given the age of the halo, $t_{\mathrm{b}}$ and the main sequence lifetime as a function of the mass in the main sequence (Iben \& Laughlin 1989) we know which stars have had time enough to become white dwarfs, and given a set of cooling sequences (Salaris et al. 2000) and the initial to final mass relationship (Iben \& Laughlin 1989), which are their luminosities.

The velocity distribution has been modeled according to a Gaussian law (Binney \& Tremaine 1987):

$$
f\left(v_{r}, v_{t}\right)=\frac{1}{(2 \pi)^{3 / 2}} \frac{1}{\sigma_{r} \sigma_{t}^{2}} \exp \left[-\frac{1}{2}\left(\frac{v_{r}^{2}}{\sigma_{t}^{2}}+\frac{v_{t}^{2}}{\sigma_{t}^{2}}\right)\right]
$$

where $\sigma_{r}$ and $\sigma_{t}-$ the radial and the tangential velocity dispersion, respectively - are related by the following expression:

$\sigma_{t}^{2}=\frac{V_{\mathrm{c}}^{2}}{2}+\left[1-\frac{r^{2}}{a^{2}+r^{2}}\right] \sigma_{r}^{2}+\frac{r}{2} \frac{\mathrm{d}\left(\sigma_{r}^{2}\right)}{\mathrm{d} r}$ 
Table 1. The different IMFs used in this paper.

\begin{tabular}{|c|c|c|}
\hline IMF & $\phi(M)$ & Reference \\
\hline Standard & $\begin{cases}\left(M / M_{\odot}\right)^{-1.2} & \text { for } M<1.0 M_{\odot} \\
\left(M / M_{\odot}\right)^{-2.7} & \text { for } 1.0 M_{\odot}<M<10.0 M_{\odot} \\
0.4\left(M / M_{\odot}\right)^{-2.3} & \text { for } M>10.0 M_{\odot}\end{cases}$ & Scalo (1998) \\
\hline CSM1 & $\left\{\begin{array}{c}\exp \left[-\left(M_{\text {cut }} / M\right)^{\beta_{1}}\left(M / M_{\odot}\right)^{\beta_{2}}\right] \\
M_{\text {cut }}=2.0 M_{\odot}, \beta_{1}=2.2 \mathrm{y} \beta_{2}=5.15\end{array}\right.$ & Chabrier et al. (1996) \\
\hline CSM2 & $\left\{\begin{array}{c}\exp \left[-\left(M_{\text {cut }} / M\right)^{\beta_{1}}\left(M / M_{\odot}\right)^{\beta_{2}}\right] \\
M_{\text {cut }}=2.7 M_{\odot}, \beta_{1}=2.2 \text { y } \beta_{2}=5.75\end{array}\right.$ & Chabrier et al. (1996) \\
\hline AL & $\left\{\begin{array}{l}\exp \left[1-\frac{1}{2 \sigma_{2}}\left(\log \left(\frac{M}{M}{ }_{\mathrm{cut}}\right)^{2}\right]\right. \\
M_{\mathrm{cut}}=2.3, \text { y } \sigma=0.44\end{array}\right.$ & Adams \& Laughlin (1996) \\
\hline
\end{tabular}

which, to a first approximation, leads to $\sigma_{r}=\sigma_{t}=V_{\mathrm{c}} / \sqrt{2}$. - see, for instance, Binney \& Tremaine (1987). For the calculations reported here we have adopted a circular velocity $V_{\mathrm{c}}=220 \mathrm{~km} \mathrm{~s}^{-1}$. From these velocities we obtain the heliocentric velocities by adding the velocity of the LSR $v_{\mathrm{LSR}}=-220 \mathrm{~km} \mathrm{~s}^{-1}$ and the peculiar velocity of the sun: $\left(U_{\odot}, V_{\odot}, W_{\odot}\right)=(10.0,15.0,8.0) \mathrm{km} \mathrm{s}^{-1}$ (Dehnen \& Binney 1998). Since white dwarfs usually do not have determinations of the radial component of the velocity, when needed for the observational comparison the radial velocity is eliminated. Moreover, we only consider stars with tangential velocities in the range $250 \lesssim v_{t} \lesssim 750 \mathrm{~km} \mathrm{~s}^{-1}$. Stars with velocities smaller than $250 \mathrm{~km} \mathrm{~s}^{-1}$ would not be considered as halo members, whereas stars with velocities larger than $750 \mathrm{~km} \mathrm{~s}^{-1}$ would have velocities exceeding 1.5 times the escape velocity, which we obtain from Binney \& Tremaine (1987):

$v_{\mathrm{e}}^{2}=2 V_{\mathrm{c}}^{2}\left[1+\ln \left(r_{*} / r\right)\right]$

where $r_{*} \simeq 41 \mathrm{kpc}$ is the radius of the galactic halo.

To build the white dwarf luminosity function using the $1 / V_{\max }$ method (Schmidt 1968) a smaller sample of white dwarfs must be culled from the original sample and to do this a set of restrictions in visual magnitude and proper motion must be adopted. The restriction in magnitude will be discussed in Sect. 3 below. Regarding the proper motion cut we have chosen $\mu \geq 0.16^{\prime \prime} \mathrm{yr}^{-1}$ as in Oswalt et al. (1996) and in García-Berro et al. (1999). Besides, the $1 / V_{\max }$ method requires that all the objects belonging to the restricted sample must have known parallaxes. This, in turn, means that all the white dwarfs belonging to this sample are within a sphere of radius of roughly 200 pc centered on the location of the sun.

\subsection{Microlensing events towards the LMC}

In order to produce a set of microlensing events towards the LMC we also need to simulate the characteristics of the white dwarf population towards the LMC. For this purpose we generate the three galactic coordinates $(r, l, b)$ of the white dwarfs of the galactic halo inside a small pencil of $4^{\circ} \times 4^{\circ}$ centered in the LMC location, $(l, b)=\left(280^{\circ},-33^{\circ}\right)$. The $l$ and $b$ distributions are practically uniform in this small window. The radial coordinate is always smaller than the outer limit of the halo $(r<41 \mathrm{kpc})$ and according to the radial distribution

$\rho=\rho_{0}\left(R_{\odot} / r\right)^{\gamma}$

where $\gamma=3.4-$ see, however, Sect. 3.7. We have chosen this distribution instead of that of Eq. (1) because in this way the number of microlensing events is maximized. In fact the mass distribution of microlenses does not necessarily follow the mass distribution of the luminous halo. However, in a first step (see Sect. 3.6 below), we have normalized the density of white dwarfs obtained from this distribution to the white dwarf density of the local neighborhood, $n \sim 9.0 \times 10^{-6} \mathrm{pc}^{-3}$ for $\log \left(L / L_{\odot}\right) \gtrsim-3.5$ (Torres et al. 1998). The velocity dispersions in this case are determined from Marković \& Sommer-Larsen (1996). For the radial velocity dispersion we have:

$\sigma_{r}^{2}=\sigma_{0}^{2}+\sigma_{+}^{2}\left[\frac{1}{2}-\frac{1}{\pi} \arctan \left(\frac{r-r_{0}}{l}\right)\right]$

where $\sigma_{0}=80 \mathrm{~km} \mathrm{~s}^{-1}, \sigma_{+}=145 \mathrm{~km} \mathrm{~s}^{-1}, r_{0}=10.5 \mathrm{kpc}$ and $l=5.5 \mathrm{kpc}$. The tangential dispersion is given by:

$\sigma_{t}^{2}=\frac{1}{2} V_{\mathrm{c}}^{2}-\left(\frac{\gamma}{2}-1\right) \sigma_{r}^{2}+\frac{r}{2} \frac{\mathrm{d} \sigma_{r}^{2}}{\mathrm{~d} r}$

where

$r \frac{\mathrm{d} \sigma_{r}^{2}}{\mathrm{~d} r}=-\frac{1}{\pi} \frac{r}{l} \frac{\sigma_{+}^{2}}{1+\left[\left(r-r_{0}\right) / l\right]^{2}}$.

In order to decide which white dwarf could potentially produce a microlensing event a magnitude cut must be adopted. If the white dwarf is brighter than this limiting magnitude it could be potentially detected and, consequently, would not be a genuine microlensing event. We have explored a wide range of possibilities, namely $m_{\mathrm{v}}^{\text {cut }}=17.5 \mathrm{mag}, 22.5 \mathrm{mag}$ and $27.5 \mathrm{mag}$, but, as a fiducial value, we have taken $m_{\mathrm{V}}^{\text {cut }}=17.5 \mathrm{mag}$, which is the value adopted by Alcock et al. (2000). We also need to simulate the population of stars of the LMC itself. In order to produce 
such a population we distribute the number of monitored point sources $\left(\sim 1.2 \times 10^{7}\right)$ according to the LMC model of Gyuk et al. (2000). That is, we have adopted a scale lenght, $R_{\mathrm{d}}=1.57 \mathrm{kpc}$, a scale height $z_{\mathrm{d}}=0.3 \mathrm{kpc}$, an inclination angle $i=30^{\circ}$, a distance $L=50 \mathrm{kpc}$, a position angle, $\phi=170^{\circ}$, and a tangential heliocentric velocity, $v_{\mathrm{t}}=336 \mathrm{kms}^{-1}$. The next step is to determine if there exists a white dwarf in the line of sight to a given simulated star in the LMC. We consider a white dwarf to be responsible of a microlensing event if the angular distance between the white dwarf and the monitored star is smaller than the Einstein radius $\theta_{\mathrm{E}}=R_{\mathrm{E}} / D_{\mathrm{OL}}$, where $R_{\mathrm{E}}$ is the Einstein radius and $D_{\mathrm{OL}}$ is the distance between the observer and the lens. This, of course, can happen at any time during the total monitoring period of $5.7 \mathrm{yr}$, due to the proper motion of the lenses, which is obtained from the previous equations.

The optical depth is obtained, following Alcock et al. (2000), using the expression

$\tau=\frac{1}{E} \frac{\pi}{4} \sum_{i} \frac{\hat{t}_{i}}{\varepsilon\left(\hat{t}_{i}\right)}$

where $E=6.12 \times 10^{7}$ is the total exposure (in star-years), $\hat{t}_{i}$ is the Einstein ring diameter crossing time, and $\varepsilon\left(\hat{t}_{i}\right)$ is the detection efficiency. The detection efficiency has been modelled as:

$\varepsilon(\hat{t})=\left\{\begin{array}{cc}0.43 \mathrm{e}^{-\left(\ln \left(\hat{t} / T_{\mathrm{m}}\right)\right)^{3.58} / 0.87}, & \hat{t}>T_{\mathrm{m}} \\ 0.43 \mathrm{e}^{-\left|\ln \left(\hat{t} / T_{\mathrm{m}}\right)\right|^{2.34} / 11.16}, & \hat{t}<T_{\mathrm{m}}\end{array}\right.$

where $T_{\mathrm{m}}=250$ days. This expression provides a good fit to the results of Alcock et al. (2000).

\subsection{The Hubble Deep Field simulation}

For the simulation of the HDF we have distributed stars in a window of $1.2^{\circ} \times 1.2^{\circ}$ centered around $(l, b)=\left(125^{\circ}, 55^{\circ}\right)$. The radial distribution is, again, according to Eq. (5) within the outer halo limit. The velocities are consequently drawn from Eqs. (2) and (6) to (8). This simulation has been also normalized to the local density given by the halo simulation.

\subsection{The EROS simulation}

Finally, for the simulation of the EROS results we have distributed stars in a window of $190^{\circ 2}$ in the Southern Galactic Hemisphere $\left(-79^{\circ}<b_{\text {gal }}<-48^{\circ}\right)$, in the following strips along the $\alpha$ coordinate, $\Delta \delta=1.4^{\circ}$ wide: $22^{\mathrm{h}} 16^{\mathrm{min}}<\alpha<3^{\mathrm{h}} 44^{\mathrm{min}}$ at $\delta=-44^{\circ} 45^{\prime}, 23^{\mathrm{h}} 31^{\mathrm{min}}<\alpha<1^{\mathrm{h}} 34^{\mathrm{min}}$ at $\delta=-40^{\circ} 09^{\prime}$ and $22^{\mathrm{h}} 24^{\mathrm{min}}<\alpha \leq 3^{\mathrm{h}} 28^{\mathrm{min}}$ at $\delta=-38^{\circ} 45^{\prime}$, and $187^{\circ 2}$ in the Northern Galactic Hemisphere $\left(41^{\circ}<b_{\text {gal }}<59^{\circ}\right)$ with $10^{\mathrm{h}} 57^{\mathrm{min}}<\alpha \leq 13^{\mathrm{h}} 23^{\mathrm{min}}$ at $\delta=-12^{\circ}$ and $10^{\mathrm{h}} 57^{\mathrm{min}}<\alpha<$ $12^{\mathrm{h}} 53^{\mathrm{min}}$ at $\delta=-4^{\circ} 36^{\prime}$. The radial distribution and the velocity distribution are, again, the same used for the HDF simulation. Also, the density of white dwarfs has been normalized to the local density given by the halo simulation.

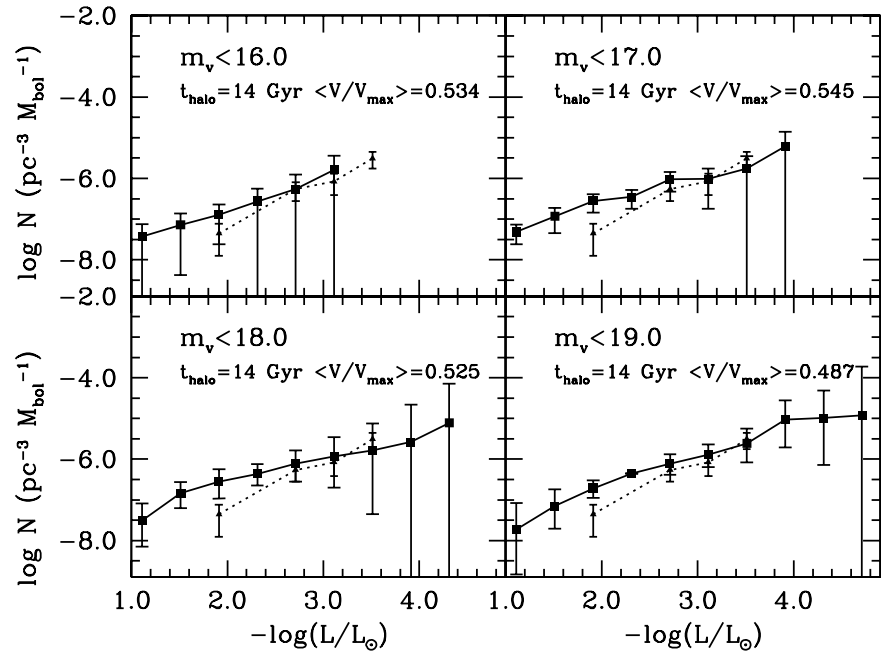

Fig. 1. Luminosity function of halo white dwarfs for several limiting magnitudes.

\section{Results}

\subsection{The halo white dwarf luminosity function}

One of the most serious problems that is found when determining the observational white dwarf luminosity function of halo white dwarfs is that the real limiting magnitude used in these studies is highly uncertain. We have conducted a series of simulations with different limiting magnitudes to determine which would be the limiting magnitude able to reproduce the observational luminosity function. In Fig. 1 we show the white dwarf luminosity functions obtained for several limiting magnitudes (16, 17, 18 and 19 mag) as solid lines. The luminosity function of Torres et al. (1998) is shown as a dashed line for comparison purposes. Each panel is clearly marked with its corresponding limiting magnitude. The adopted halo age in all the cases was 14 Gyr. Also the adopted IMF is the standard one in all four simulations. The error bars of each luminosity bin were computed according to Liebert et al. (1988): the contribution of each star to the total error budget in its bin is conservatively estimated to be the same amount that contributes to the resulting density; the partial contributions of each star in the bin are squared and then added, the final error being the square root of this value. This procedure is followed for each of the 40 realizations of the Monte Carlo simulation. After doing this the ensemble average of the dispersions is computed. Obviously the larger the magnitude limit the fainter the white dwarfs we detect. If we disregard as non-significant the bin in which we only detect on average one white dwarf we see that the limiting magnitude that best reproduces the luminosity function of Torres et al. (1998) is $m_{\mathrm{V}}^{\lim }=17 \mathrm{mag}$. Therefore for the rest of the simulations we adopt this value. To detect the cut-off of the luminosity function a limiting magnitude of 25 mag should be adopted. Since the heliocentric velocity of halo white dwarfs is considerable, the proper motion cut plays a limited role. In fact the proper motion cut only affects the total number of white dwarfs in the sample but not the shape of the luminosity function. In contrast, as we have seen, this is not the case for the cut in magnitude. This is the same as to say that 


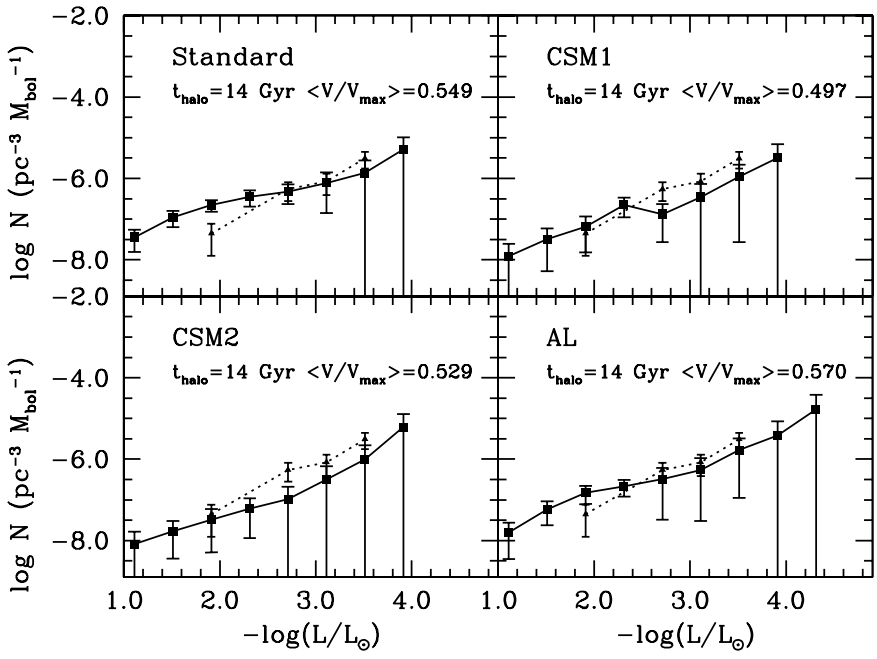

Fig. 2. Luminosity function of halo white dwarfs for several IMFs.

the proper motion cut equally affects all the luminosity bins. It is as well interesting to note here that the value of the limiting magnitude that we have found so far is in close agreement with the cut adopted by Alcock et al. (2000) for the monitoring of stars within the MACHO project, which is $17.5 \mathrm{mag}$.

\subsection{The role of the IMF}

In Fig. 2 we show the luminosity functions obtained with a Salpeter-like mass function, the two log-normal IMFs proposed by Chabrier et al. (1996) - CSM1 and CSM2, respectively and the IMF of Adams \& Laughlin (1996) - AL. As this figure clearly shows, the derived luminosity functions are not very sensitive to the precise shape of the IMF. Moreover, the completeness, as measured by the $\left\langle V / V_{\max }\right\rangle$ method, seems to be similar in all four cases. Only in the cases CSM1 and, more apparently, in the CSM2 case is there a slight underproduction of luminous white dwarfs. However, the significance is only marginal and, therefore, to constrain the IMF of the galactic halo using intrinsically bright white dwarfs deeper surveys are needed. We will come back to this issue when studying the HDF simulation in Sect. 3.4 below.

As previously stated in Sect. 2.1, all the luminosity functions obtained here have been normalized to the local density of halo white dwarfs obtained by Torres et al. (1998), $n \sim 9.0 \times 10^{-6} \mathrm{pc}^{-3}$ for $\log \left(L / L_{\odot}\right) \gtrsim-3.5$, which for a typical value of the mass of white dwarfs $\left(\simeq 0.6 M_{\odot}\right)$ corresponds to a density of halo baryonic matter in the form of white dwarfs of $\sim 7.2 \times 10^{-6} M_{\odot} \mathrm{pc}^{-3}$. However, from our simulations we can derive the total density of baryonic matter in the galactic halo within $300 \mathrm{pc}$ from the sun. We obtain $\rho_{0}=2.2 \times 10^{-4} M_{\odot} \mathrm{pc}^{-3}$ for the standard IMF, $2.0 \times 10^{-3} M_{\odot} \mathrm{pc}^{-3}$ for the CSM1 case, $1.1 \times 10^{-2} M_{\odot} \mathrm{pc}^{-3}$ for the CSM2, and $2.7 \times 10^{-3} M_{\odot} \mathrm{pc}^{-3}$ for the AL simulation. These values, in turn, correspond to a fraction $\eta$ of baryonic dark matter of $0.03,0.25,1.40$ and 0.33 , respectively. The differences between all the IMFs analyzed here are considerable. For instance, for the CSM2 case we would have more matter than needed, whereas the CSM1 and AL mass functions would lock a sizeable fraction of dark matter in the

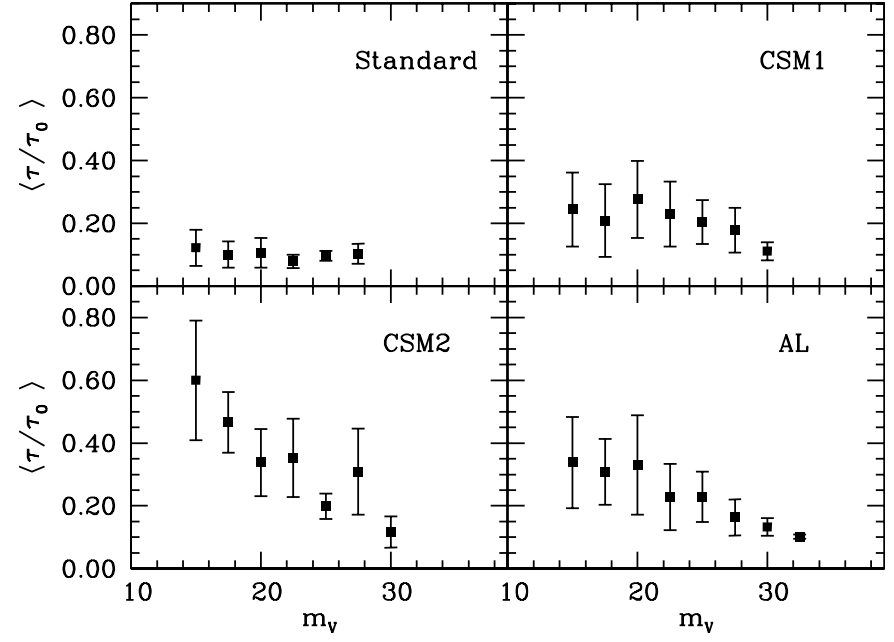

Fig. 3. Microlensing optical depth towards the LMC as a function of the limiting magnitude for several IMFs.

form of main sequence stars, stellar remnants and in the corresponding ejected mass. Finally the standard IMF only allows for a modest $3 \%$ of the required dark matter. In this case, moreover, roughly $1 / 3$ of the stellar content corresponds to white dwarfs.

\subsection{Microlenses towards the $L M C$}

In Fig. 3 we show the microlensing optical depth towards the LMC obtained in our simulations normalized to the value derived by Alcock et al. (2000), $\tau_{0}=1.2 \times 10^{-7}$, as a function of the magnitude cut for detection of white dwarfs (see section Sect. 3.1). As it can be seen in this figure the contribution of white dwarfs to the optical depth depends sensitively on the adopted IMF. For the standard IMF we derive a contribution of roughly $10 \%$, no matter what the magnitude cut is, whereas for the biased IMFs we obtain contributions which are, typically, of $23 \%$ for the CSM1 case, $35 \%$ for the CSM2 case, and $22 \%$ for the AL simulation. These values are relatively constant for large enough magnitude cuts and, hence, for realistic values of the magnitude cut they can be considered as safe. Conversely, the microlensing optical depth is a robust indicator of the density of the microlenses. It is important to realize that none of the adopted IMFs is able to reproduce satisfactorily the value found by the MACHO team, in spite of the very extreme assumptions adopted for deriving the log-normal biased IMFs, which were especially tailored to reproduce the microlensing results.

Nevertheless the information that can be derived from our Monte Carlo simulations is far more complete. A summary of the results can be found in Table 2, where we show the number of microlensing events, the average mass of the microlenses, the average proper motion and distance, the average tangential velocity of the microlenses, the corresponding Einstein crossing times and, finally, the contribution to the optical depth, all of them for three selected magnitude cuts. As it can be seen in Table 2 none of the IMFs is able to reproduce the observed number of microlensing events (13 to 17 , depending on the 
Table 2. Summary of the results obtained for the simulation of microlenses towards the LMC for an age of the halo of 14 Gyr, different model IMFs, and several magnitude cuts.

\begin{tabular}{|c|c|c|c|c|c|c|c|c|c|c|c|c|}
\hline & \multicolumn{3}{|c|}{ Standard } & \multicolumn{3}{|c|}{ CSM1 } & \multicolumn{3}{|c|}{ CSM2 } & \multicolumn{3}{|c|}{$\mathrm{AL}$} \\
\hline Magnitude & 17.5 & 22.5 & 27.5 & 17.5 & 22.5 & 27.5 & 17.5 & 22.5 & 27.5 & 17.5 & 22.5 & 27.5 \\
\hline$\left\langle N_{\mathrm{WD}}\right\rangle$ & $0 \pm 1$ & $0 \pm 1$ & $0 \pm 1$ & $2 \pm 4$ & $1 \pm 3$ & $1 \pm 2$ & $7 \pm 3$ & $6 \pm 4$ & $2 \pm 2$ & $5 \pm 5$ & $3 \pm 3$ & $1 \pm 1$ \\
\hline$\langle m\rangle\left(M / M_{\odot}\right)$ & 0.564 & 0.568 & 0.556 & 0.590 & 0.582 & 0.601 & 0.598 & 0.613 & 0.595 & 0.622 & 0.636 & 0.614 \\
\hline$\langle\mu\rangle\left(" \mathrm{yr}^{-1}\right)$ & 0.016 & 0.014 & 0.012 & 0.030 & 0.024 & 0.010 & 0.093 & 0.035 & 0.021 & 0.035 & 0.030 & 0.012 \\
\hline$\langle d\rangle(\mathrm{kpc})$ & 3.09 & 3.14 & 5.05 & 1.53 & 2.12 & 6.19 & 0.51 & 1.50 & 3.04 & 1.63 & 1.65 & 5.10 \\
\hline$\left\langle V_{\mathrm{tan}}\right\rangle\left(\mathrm{km} \mathrm{s}^{-1}\right)$ & 235 & 207 & 285 & 222 & 243 & 291 & 224 & 251 & 308 & 272 & 232 & 293 \\
\hline$\left\langle\hat{t}_{\mathrm{E}}\right\rangle(\mathrm{d})$ & 61.3 & 75.8 & 89.3 & 36.3 & 52.6 & 70.8 & 21.7 & 41.7 & 70.9 & 28.8 & 42.7 & 69.2 \\
\hline$\left\langle\tau / \tau_{0}\right\rangle$ & 0.101 & 0.079 & 0.102 & 0.209 & 0.230 & 0.177 & 0.466 & 0.353 & 0.308 & 0.308 & 0.228 & 0.163 \\
\hline
\end{tabular}

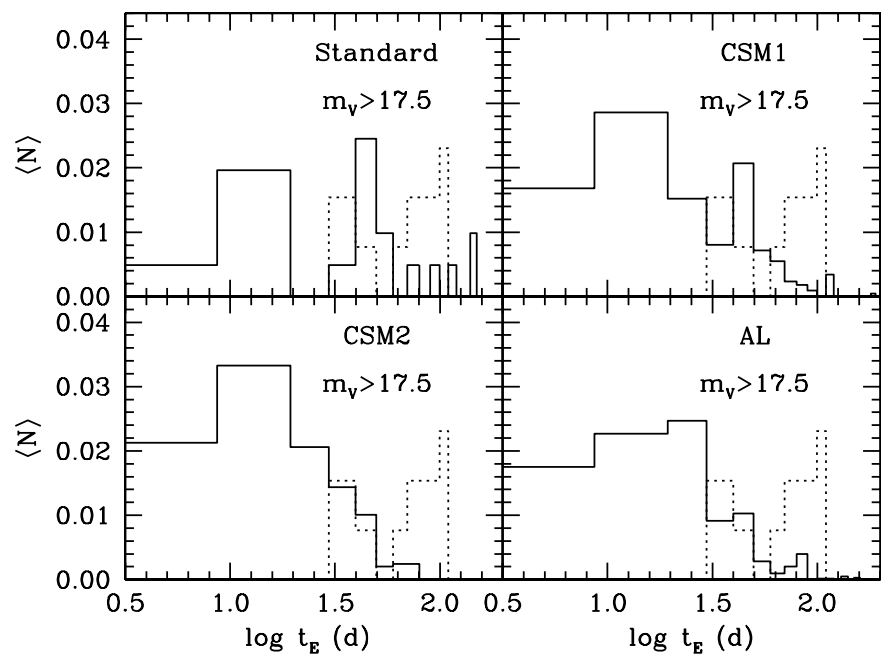

Fig. 4. Distribution of Einstein crossing times for the microlensing events towards the LMC of the 40 independent realizations of the simulated population (solid lines) for several IMFs, and of the observational data (dashed lines).

selection criteria) found by Alcock et al. (2000). Even in the case of the CSM2 simulation, which corresponds to the most extreme assumption on the IMF, we obtain only $7 \pm 3$ microlensing events in the best of the cases. This explains why the contribution to the microlensing optical depth of the white dwarf population is within the range $10 \%$ to $35 \%$, depending on the adopted IMF, suggesting that a sizeable fraction of the microlensing events could be either self-lensing in the LMC (Salati et al. 1999) or due to background objects (Green \& Jedamzik 2002).

Further important information that can be readily obtained from the simulations presented so far is the distribution of Einstein crossing times. Such distributions for the 40 independent realizations are shown in Fig. 4 for the four cases studied here as solid lines. We have chosen the brighter of our magnitude cuts in order to allow for a larger number of events. Note, however, that this magnitude cut is exactly the same adopted by the MACHO team. Also shown, as dashed lines, are the distribution of Einstein crossing times obtained by the MACHO team. All the distributions have been normalized to unit area and, hence, are frequency distributions. The average Einstein crossing times for each of the simulations can be also found in Table 2. As it can be seen in Fig. 4 the MACHO team detects microlensing events with larger durations than those obtained in the simulations of the log-normal IMFs of Chabrier et al. (1996) and Adams \& Laughlin (1996). Although the statistics is poor, we can compare the average crossing times obtained here with the average crossing time obtained by the MACHO team which is of $76 \pm 23$ days. These average crossing times are respectively $\sim 61,36,22$ and 29 days. In all the cases the typical standard deviation is of about 15 days. We emphasize here that these averages are the result of an ensemble of forty simulations. Clearly, biased mass functions yield considerably smaller average Einstein crossing times than those of the MACHO team, whereas a standard IMF yields a value which is actually within the $1 \sigma$ error bars of the observed one. Since $\left\langle t_{\mathrm{E}}\right\rangle^{2}$ is an indicator of the mass of the lens it follows that the average mass of the lenses for biased mass functions must be different of that of the standard one. And this is, indeed, the case as it can be seen in Table 2. This behavior is not surprising since the three log-normal mass functions partially inhibit the formation of low mass white dwarfs. In all these three cases the observed average Einstein crossing time is beyond the $1 \sigma$ error bars ( $\sim 15$ days). Hence, although this mass functions yield a significantly higher number of microlensing events they have considerably smaller Einstein crossing times.

Another interesting fact which results from a careful study of Table 2 has to do with the tangential velocities of the simulated microlensing events. As it can be seen the average tangential velocity of the microlenses is typical of the halo population, with values close to the canonical one of $220 \mathrm{~km} \mathrm{~s}^{-1}$. But, on the contrary, the average distances of the microlenses do depend on the adopted IMF. For instance, the microlenses produced by the standard IMF are located, on average, at larger distances $(3.14 \mathrm{kpc})$, than those of the biased IMFs $(2.12,1.50$ and $1.65 \mathrm{kpc}$, respectively). In fact, the larger the adopted mass cut of the IMF is (see Table 1), the closer the microlenses are. This, in turn, translates directly into the average proper motions of the lenses, since, as already mentioned, the tangential velocities of the lenses do not differ much in the simulations reported here. Finally, as expected, the average mass of the microlenses differs considerably in all the cases studied here. For the standard IMF we obtain a mass of $0.56 M_{\odot}$, close to the canonical average mass of disk white dwarfs. For the biased IMFs we obtain values of $0.58 M_{\odot}, 0.61 M_{\odot}$ and $0.64 M_{\odot}$, respectively, which reflect, as already pointed out, the adopted mass cut for the IMF.

In Fig. 5 we show the relative distribution (normalized to unit area) in magnitudes of the whole population of white 
Table 3. Summary results of the microlensing events towards the LMC for a limiting magnitude of $m_{\mathrm{V}}=17.5$ mag and different halo ages and IMFs.

\begin{tabular}{lcccccc}
\hline \hline & \multicolumn{3}{c}{ Standard } & & CSM1 \\
\hline Age & $12 \mathrm{Gyr}$ & $14 \mathrm{Gyr}$ & $16 \mathrm{Gyr}$ & $12 \mathrm{Gyr}$ & $14 \mathrm{Gyr}$ & $16 \mathrm{Gyr}$ \\
\cline { 2 - 7 }$\left\langle N_{\mathrm{WD}}\right\rangle$ & $0 \pm 1$ & $0 \pm 1$ & $0 \pm 1$ & $1 \pm 3$ & $2 \pm 4$ & $2 \pm 4$ \\
$\langle m\rangle\left(M / M_{\odot}\right)$ & 0.600 & 0.565 & 0.564 & 0.588 & 0.590 & 0.586 \\
$\langle\mu\rangle\left({ }^{\prime \prime} \mathrm{yr}^{-1}\right)$ & 0.029 & 0.016 & 0.022 & 0.032 & 0.030 & 0.028 \\
$\langle d\rangle(\mathrm{kpc})$ & 1.19 & 3.09 & 2.55 & 1.59 & 1.53 & 1.78 \\
$\left\langle V_{\mathrm{tan}}\right\rangle\left(\mathrm{km} \mathrm{s}^{-1}\right)$ & 260 & 235 & 274 & 243 & 222 & 238 \\
$\left\langle\hat{t}_{\mathrm{E}}\right\rangle(\mathrm{d})$ & 55.3 & 61.4 & 44.3 & 37.9 & 36.3 & 33.5 \\
$\left\langle\tau / \tau_{0}\right\rangle$ & 0.110 & 0.101 & 0.090 & 0.178 & 0.209 & 0.196 \\
\hline & & & & & & \\
\hline & & $\mathrm{CSM} 2$ & & & $\mathrm{AL}$ & \\
\hline Age & $12 \mathrm{Gyr}$ & $14 \mathrm{Gyr}$ & $16 \mathrm{Gyr}$ & $12 \mathrm{Gyr}$ & $14 \mathrm{Gyr}$ & $16 \mathrm{Gyr}$ \\
$\left\langle N_{\mathrm{WD}}\right\rangle$ & $8 \pm 3$ & $7 \pm 3$ & $7 \pm 3$ & $5 \pm 5$ & $5 \pm 5$ & $3 \pm 3$ \\
$\langle m\rangle\left(M / M_{\odot}\right)$ & 0.620 & 0.598 & 0.589 & 0.642 & 0.622 & 0.650 \\
$\langle\mu\rangle\left({ }^{\prime \prime} \mathrm{yr}^{-1}\right)$ & 0.039 & 0.093 & 0.041 & 0.043 & 0.035 & 0.026 \\
$\langle d\rangle\left(\mathrm{kpc}^{-1}\right)$ & 1.24 & 0.51 & 1.04 & 1.07 & 1.63 & 1.66 \\
$\left\langle V_{\mathrm{tan}}\right\rangle\left(\mathrm{km} \mathrm{s}^{-1}\right)$ & 230 & 224 & 202 & 219 & 272 & 203 \\
$\left\langle\hat{t}_{\mathrm{E}}\right\rangle(\mathrm{d})$ & 27.0 & 21.7 & 20.9 & 38.1 & 28.8 & 48.4 \\
$\left\langle\tau / \tau_{0}\right\rangle$ & 0.491 & 0.466 & 0.415 & 0.323 & 0.308 & 0.310 \\
\hline
\end{tabular}

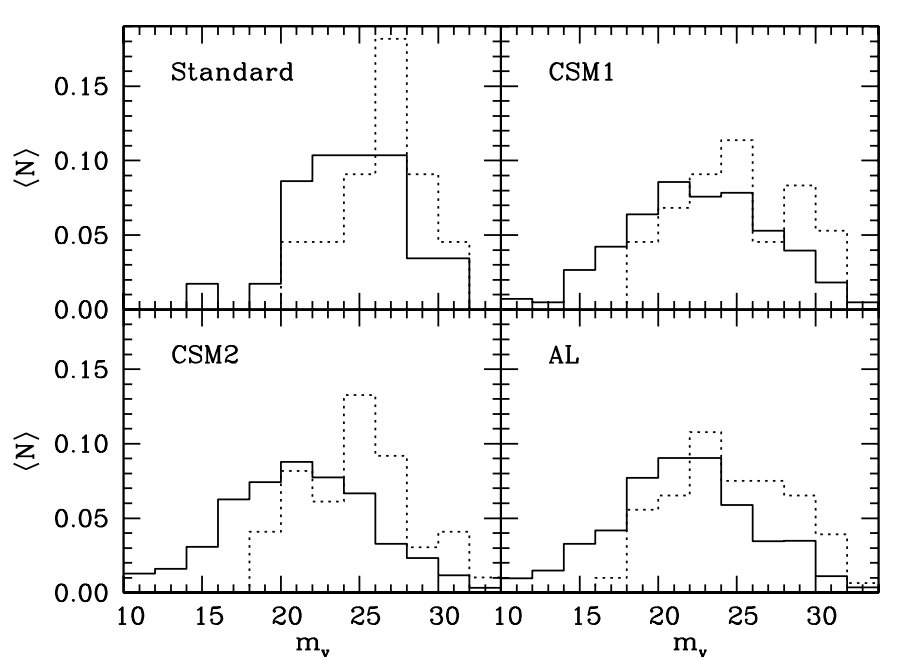

Fig. 5. Distribution of the whole white dwarf population - solid lines - and of the white dwarfs responsible for microlensing events towards the LMC - dashed lines - as a function of their visual magnitude for several IMFs.

dwarfs - solid lines - and of those white dwarfs responsible of the microlensing events - dashed lines. Again, the distributions have been normalized to unit area. As it can be seen in this figure, most of the white dwarfs have apparent magnitudes brighter than 25 mag and, hence, the magnitude cut only plays a significant role in the case in which the magnitude cut is $27.5 \mathrm{mag}$, which is not very realistic. Therefore the results quoted in Table 2 do not depend much on the adopted magnitude cut.

Finally, we have analyzed the dependence of these results on the adopted age of the halo. We have chosen halo ages of 12, 14, and 16 Gyr. In all these simulations we have adopted the same magnitude cut $m_{\mathrm{V}}^{\text {cut }}=17.5 \mathrm{mag}$. The results obtained in these sets of simulations are shown in Table 3. From an analysis of this table we see that the results do not depend much on the adopted halo age. In particular the number of microlensing events remains almost constant regardless of the precise value of the age of the halo. Also, and most importantly, the same behavior can be observed for the computed microlensing optical depth. Thus, the actual values of the number of microlensing events and of the microlensing optical depth derived here are quite robust.

\subsection{The Hubble Deep Field}

The deepest optical images obtained up to now are those of the Hubble Deep Field. In spite of the very small area surveyed by the $\mathrm{HDF}\left(\simeq 4.4 \operatorname{arcmin}^{2}\right)$, the limiting magnitude of $m_{\mathrm{V}}^{\lim } \sim 28 \mathrm{mag}$ allows to probe a large volume. Therefore, these results complement those of the MACHO team and can provide us with relevant (and very valuable) information about the halo of the Galaxy. Nevertheless, the results obtained so far by different authors are not concluding. For instance Flynn et al. (1996) studied the HDF north and were able to distinguish between stars and galaxies down to magnitudes as faint as $I=26$. Their selection criteria in the search of white dwarfs are summarized in the first row of Table 4. They did not find any object with $V-I>1.8$ although their results were consistent with an upper limit of 3 white dwarfs. Later Méndez et al. (1996) detected 6 faint objects $(V \geq 25)$ with color indexes in the range $-0.5<B-V<0.5$ or, equivalently, $0<V-I<1.2$. However, these objects could be as well non resolved galaxies. Finally, the most plausible analysis of the HDF is that of Ibata et al. (1999). These authors determined the proper motions of the objects in the HDF from two epoch observations. The time baseline was 2 yr. In this way they were able to discriminate between nearby objects and galaxies. The maximum number of white dwarfs found by Ibata et al. (1999) is 4. However, one of these identifications corresponds to a re-discovery of a previously known white dwarf, whereas the rest of the 


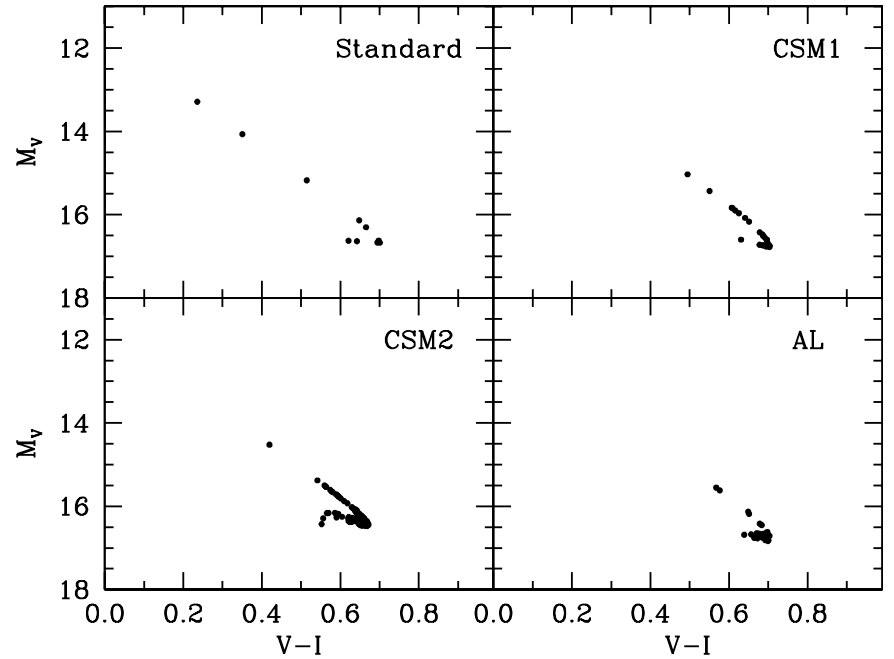

Fig. 6. Theoretical color-magnitude diagram of a typical Monte Carlo realization of the HDF for several IMFs.

Table 4. Summary of the HDF results. The authors, the selection criteria and number of objects are indicated.

\begin{tabular}{|c|c|c|}
\hline Author & Selection Criteria & $N_{\text {Obj }}$ \\
\hline Flynn et al. (1996) & $\begin{array}{c}24.63<I<26.30 \\
V-I>1.8\end{array}$ & $<3$ \\
\hline Méndez et al. (1996) & $\begin{array}{c}25 \leq V \leq 30 \\
-0.5<B-V<0.5 \\
0<V-I<1.2\end{array}$ & $\sim 6$ \\
\hline Ibata et al. (1999) & $\begin{array}{c}27<V<28 \\
-0.2<V-I<1.0\end{array}$ & 4 \\
\hline
\end{tabular}

candidates still need spectroscopic follow-up observations. It is, thus, interesting to study which would be the predictions of our Monte Carlo simulator for different IMFs.

In Fig. 6 the theoretical color-magnitude diagram of a typical Monte Carlo realization of the HDF is shown. As it can be seen in this figure most white dwarfs populate the coolest and reddest portion of the cooling isochrone. This is a consequence of the fact that the characteristic cooling times increase considerably at low luminosities due to both the release of latent heat upon crystallization and of gravitational energy due to carbon-oxygen separation (Isern et al. 2000). The characteristic " $z$ "-shaped feature at the lowest luminosities is due to the contribution of massive white dwarfs and not to the blue turn reported by Hansen (1999) - see Isern et al. (1998) and Salaris et al. (2000) for a comprehensive explanation of this feature which our cooling sequences reproduce well but for larger ages of the Galactic halo. Note as well that for biased mass functions the contribution of low mass stars has been suppressed. This, in turn, implies that these mass functions should provide a very small number of white dwarfs with moderately high luminosities (Isern et al. 1998) and, indeed, this is the case. Of course for larger mass cuts the number of white dwarfs on the hot portion of the cooling isochrone should be smaller and, hence, this is

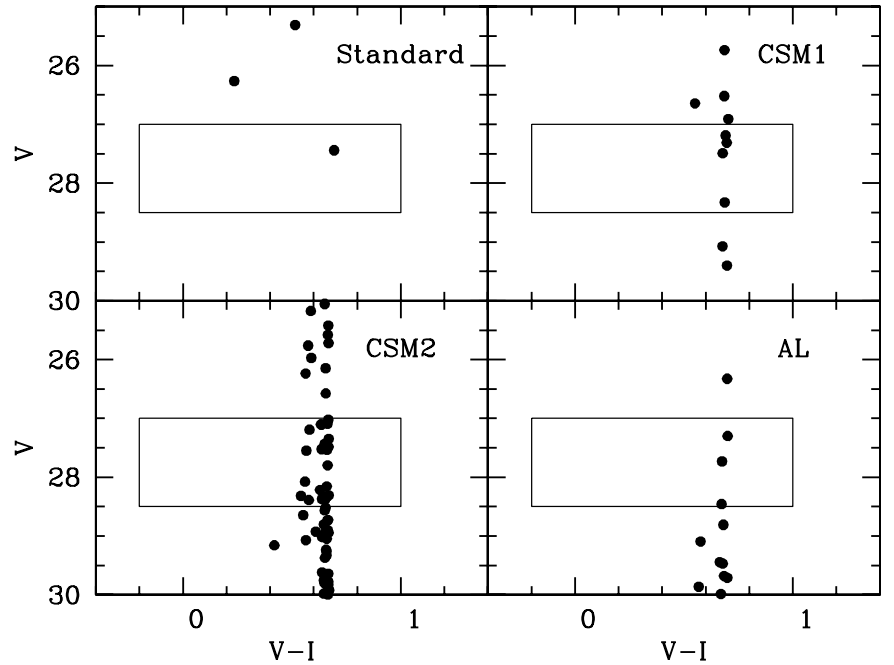

Fig. 7. Simulation of the HDF. The box represents the observational selection criteria of Ibata et al. (1999).

the reason why in the AL case and the CSM2 simulation white dwarfs tend to concentrate at the hook of the cooling isochrone.

In Fig. 7a typical realization of the simulation of the HDF is shown. In this figure the box represents the observational selection criteria of Ibata et al. (1999), as shown in Table 4. Since for biased mass functions most white dwarfs tend to be concentrated at the hook of the cooling isochrone and they are located at very different distances the result is that for these cases white dwarfs are distributed along an almost vertical strip, which is located at the position of the hook, $V-I \simeq 0.6$. This could be a potential way to test the IMF of the Galactic halo in addition to the total number of white dwarfs found in the field, since, as expected, biased mass functions provide a considerably larger number of white dwarfs when compared with the standard case.

The fraction of dark matter of a given population is usually computed according to:

$f=\frac{3 N_{\mathrm{obj}} M}{\epsilon \Omega \rho_{\mathrm{DM}}} 10^{-0.6\left(m_{\mathrm{V}}-M_{\mathrm{V}}+5\right)}$

where $N_{\mathrm{obj}}$ is the number of objects in the sample, $\rho_{\mathrm{DM}}=$ $8.0 \times 10^{-3} \mathrm{pc}^{-3}$ is the local density of halo dark matter, $M$ is the average mass of the white dwarfs in the HDF for a given simulation, $m_{\mathrm{V}}$ is the apparent magnitude cut of Ibata et al. (1999) as given in Table $4, M_{\mathrm{V}}$ is the absolute magnitude of the dimmest white dwarf in the simulated sample, $\Omega=4.4 \operatorname{arcmin}^{2}$ is the area surveyed by the HDF, and $\epsilon$ is the fraction of white dwarfs within the selection criteria over the total number of white dwarfs. This fraction has been estimated to be $\epsilon \sim 0.42$ (Ibata et al. 1999), but its real value is uncertain. Consequently, this expression does not fully take into account all the biases introduced by the selection process and, hence, the adopted value of $\epsilon$ can only be regarded as a relatively bona fide estimate. Instead, the dark matter fraction has been computed in the following way.

The expected number of objects in the sample, is given by $N_{\text {obj }}=n V_{\text {eff }}$, where $V_{\text {eff }}$ is the effective volume of the sample and $n=f \rho_{0} / M$. As shown in Eq. (11) the usual way to compute the effective volume of the sample is $V_{\text {eff }}=\epsilon \frac{1}{3} \Omega d^{3}$ where $d$ is 
Table 5. Summary of the results for the HDF obtained with our Monte Carlo simulator for the different proposed IMFs.

\begin{tabular}{lcccccc}
\hline \hline IMF & $N_{\text {Obj }}$ & $V_{\text {eff }}\left(\mathrm{pc}^{3}\right)$ & $\left\langle V / V_{\max }\right\rangle$ & $\epsilon$ & $d(\mathrm{kpc})$ & $f$ \\
\hline Standard & $2 \pm 2$ & 6360 & 0.396 & 0.69 & 3.7 & 0.04 \\
CSM1 & $4 \pm 4$ & 2051 & 0.466 & 0.79 & 2.5 & 0.20 \\
CSM2 & $30 \pm 6$ & 874 & 0.475 & 0.68 & 1.9 & 4.16 \\
AL & $3 \pm 3$ & 2180 & 0.531 & 0.80 & 2.6 & 0.14 \\
\hline
\end{tabular}

taken to be $\log d=0.2\left(m_{\mathrm{V}}-M_{\mathrm{V}}+5\right)$. Another, more accurate, way of computing the effective volume is to use the $1 / V_{\max }$ method as it follows. For each star of the sample we determine the maximum distance over which any star can contribute to the sample,

$r_{\max }=\min \left[\pi^{-1}\left(\mu / \mu_{1}\right) ; \pi^{-1} 10^{0.2\left(m_{1}-m\right)}\right]$,

and the minimum distance as well:

$r_{\min }=\max \left[\pi^{-1}\left(\mu / \mu_{\mathrm{u}}\right) ; \pi^{-1} 10^{0.2\left(m_{\mathrm{u}}-m\right)}\right]$,

where $\pi$ is the stellar parallax, $\mu$ its proper motion, $m$ the apparent magnitude, $\mu_{\mathrm{l}}$ and $\mu_{\mathrm{u}}$ are the low and high proper motion limits, if any, and $m_{\mathrm{l}}$ and $m_{\mathrm{u}}$ are the corresponding magnitude limit. The maximum volume in which a star can contribute is then

$V_{\max }=\frac{\Omega}{3}\left(r_{\max }^{3}-r_{\min }^{3}\right)$

and the number density of white dwarfs will be

$n=\sum_{i=1}^{N_{\text {obj }}} \frac{1}{V_{\max _{i}}}$.

The effective volume of the sample can be then computed as:

$V_{\text {eff }}=\frac{N_{\text {obj }}}{n}$

The value of $\left\langle V / V_{\max }\right\rangle$ is a measure of the completeness of the sample, which is related to $\epsilon$ by

$\epsilon=1-2\left|\left\langle\frac{V}{V_{\max }}\right\rangle-0.5\right|$.

We remind here that for a complete and homogeneous sample $\left\langle V / V_{\max }\right\rangle=0.5$.

The results for the HDF simulations are also shown in Table 5. For each of the cases we show the number of expected white dwarfs in the HDF, $N_{\mathrm{obj}}$, with its corresponding standard deviation, the effective volume surveyed, the value of $\left\langle V / V_{\max }\right\rangle$, the estimated completeness, the average distance of the white dwarfs in the field, and the corresponding fraction of dark matter derived from the simulations. An analysis of Table 5 reveals that the expected number of objects is roughly 3 in all the cases except for the case CSM2, for which the value of expected white dwarfs is much larger. It is interesting to compare these values with the expected number of objects if all the dark matter of the Galaxy were in the form of white dwarfs. This number ranges, depending on the selection criteria in colors and magnitudes, from 9 to 12 . Clearly even biased mass functions such as the AL and CSM1 mass functions are not able to fill the halo with white dwarfs. Moreover, for the CSM2 case even if we adopt the lower limit of 24 objects in the field the result is clearly much larger than that needed to fill all the halo with white dwarfs.

On the other hand, the effective volume surveyed by the HDF turns out to be dependent of the adopted model IMF. This would not be the case if Eq. (11) would have been adopted since in this case the effective volume surveyed only depends on the absolute magnitude of the faintest white dwarf in all the simulated samples which turns out to be $\sim 16.8 \mathrm{mag}$ - in accordance with the value adopted by Richer et al. (2000) - and on the adopted magnitude cut $\left(m_{\mathrm{V}}=28.5 \mathrm{mag}\right)$. It follows then that if this was the case, the radius of the effective volume would be in all cases $\simeq 2.2 \mathrm{kpc}$. The average proper motions obtained are

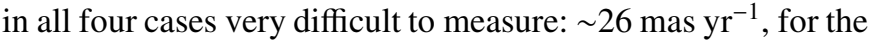

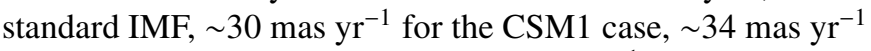

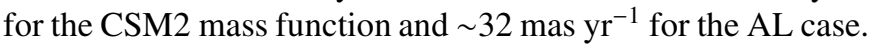

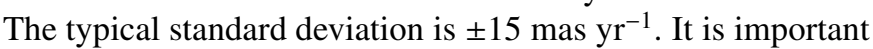
to realize that these values are in agreement with the observed value of $24 \pm 10$ mas yr$^{-1}$ (Ibata et al. 1999). The completeness of the sample is roughly the same in all four cases, whereas for the average distance of the microlenses we have significant variations. In particular for the standard IMF the average distance is considerably larger than in the rest of the simulations, being the case CSM2 an extreme case. Finally, the fraction of baryonic dark matter in the form of white dwarfs for the CSM2 case is totally incompatible with the observations, whereas in the rest of the cases is significantly smaller. For instance, for the case the CSM1 and AL mass functions the fractions are comparable, and for the standard IMF the fraction of baryonic dark matter only amounts to a modest $4 \%$. Finally it is worth noticing here that if we had used the selection criteria of Flynn et al. (1996) we would have not found any white dwarf in the HDF, whereas if we have used those of Méndez et al. (1996) we would have found $1 \pm 1$ white dwarfs in HDF, almost independently of the adopted IMF. Hence, it seems that a significant fraction of the objects found by Méndez et al. (1996) should not be white dwarfs.

\subsection{The EROS results}

The EROS team has reported very recently (Goldman et al. 2002) the results of a proper motion survey which aimed to discover faint halo white dwarfs with high proper motions. However, they did not detect any candidate halo white dwarf even if the survey was sensitive down to $V \approx 21$ and to proper motions as large as $\mu=6.0^{\prime \prime} \mathrm{yr}^{-1}$. Moreover, they found that the halo white dwarf contribution cannot exceed $5 \%$ at the $95 \%$ confidence level for objects with color index $1.0 \leq V-I \leq 1.5$. It should be noted however that this last result is dependent on the adopted model of the Galaxy. In particular Goldman et al. (2002) adopted the biased mass function CSM1 and in order to compare with the observational results they only simulated white dwarfs with magnitudes in the range $16.5 \leq M_{\mathrm{V}} \leq 18.0$ and color index within $-0.5 \leq V-I \leq 1.5$. It is therefore 


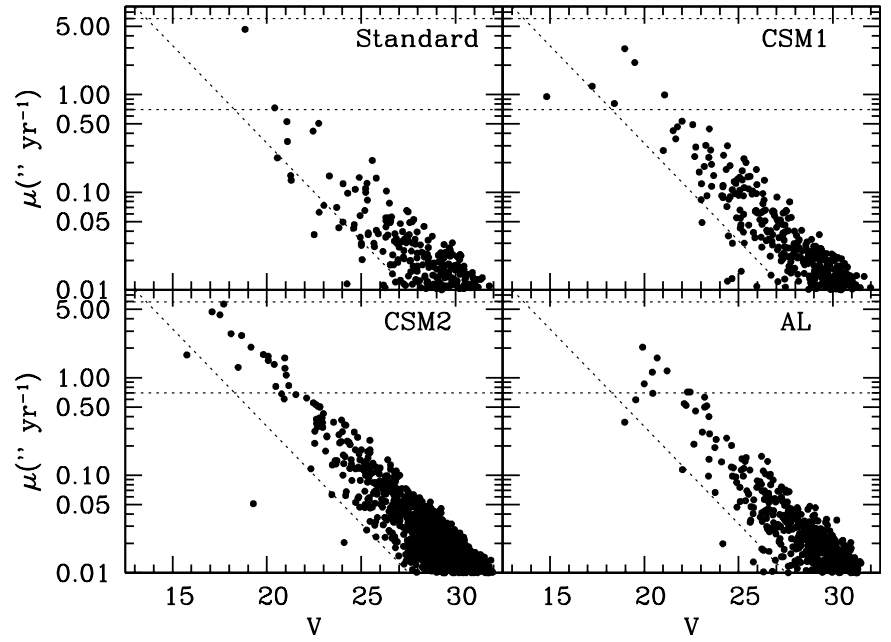

Fig. 8. Simulation of the EROS results. See text for a detailed description.

Table 6. Summary of the results for the EROS experiment obtained with our Monte Carlo simulator for the different proposed IMFs.

\begin{tabular}{lcccccc}
\hline \hline IMF & $N_{\text {obj }}$ & $V_{\text {eff }}\left(\mathrm{pc}^{3}\right)$ & $\left\langle V / V_{\max }\right\rangle$ & $\epsilon$ & $d(\mathrm{pc})$ & $f$ \\
\hline Standard & $2 \pm 1$ & 46577 & 0.210 & 0.42 & 122 & 0.01 \\
CSM1 & $6 \pm 2$ & 13256 & 0.115 & 0.32 & 81 & 0.16 \\
CSM2 & $12 \pm 4$ & 6042 & 0.150 & 0.30 & 62 & 0.55 \\
AL & $6 \pm 4$ & 10238 & 0.140 & 0.28 & 74 & 0.17 \\
\hline
\end{tabular}

necessary to extend the study to other mass functions and, moreover, to the full range of white dwarf magnitudes and colors. Additionally, since the results of the EROS team are closely connected with those of the HDF studied in Sect. 3.4 before, it is interesting to study the results obtained with our Monte Carlo simulator.

In Fig. 8 a typical Monte Carlo realization of the EROS results is shown, whereas in Table 6 the average values for the ensemble of 40 independent realizations are also shown. The entries in Table 6 are the same of Table 5, except for the distance which is expressed in pc. The selection criteria of the EROS collaboration are shown as dashed lines in Fig. 8. First, halo white dwarfs should have a reduced proper motion $H_{\mathrm{V}}=V+5 \log \pi+5>22.5$, this restriction is shown in Fig. 8 as a dashed diagonal line. Additionally they required $6.0^{\prime \prime} \mathrm{yr}^{-1}>\mu>0.7^{\prime \prime} \mathrm{yr}^{-1}$. Both limits are shown as well in Fig. 8 as a horizontal dashed lines. As it can be seen in Table 6 and in Fig. 8, in the region where the EROS experiment conducted their search for halo white dwarfs very few of them would be eventually found. In particular for the case in which a standard mass function is used only 2 of them (in the best of the cases) would be found. This is not the case for the biased mass functions CSM1 and AL for which 6 white dwarfs could be presumably found, whereas for the CSM2 case this number increases to 12. Since the EROS experiment found none the only mass function that seems to fit the observational data is the standard one. In summary, both the results of the HDF, and of the EROS experiment point towards the same conclusion: that the IMF adopted for the halo should be the standard one.

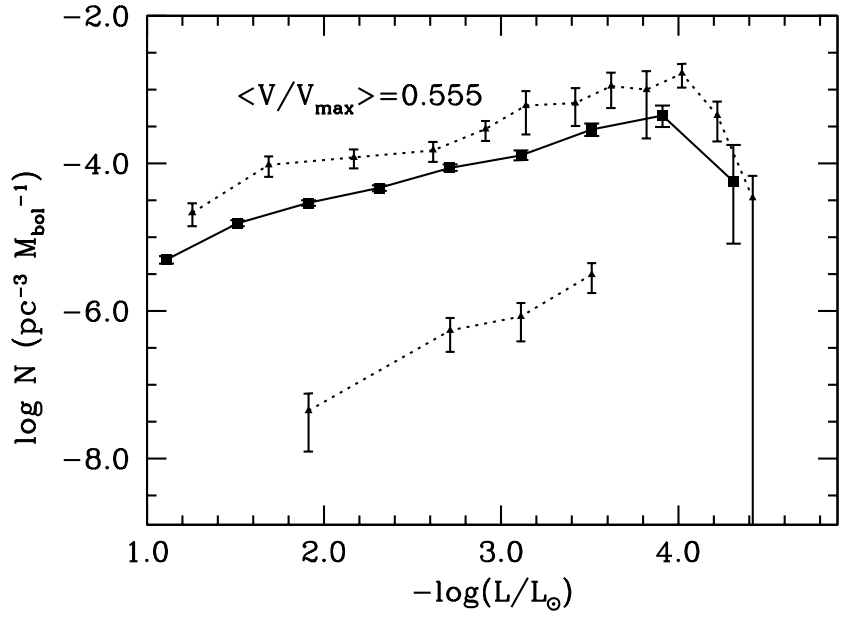

Fig. 9. The halo white dwarf luminosity function obtained when assuming that all the reported microlensing optical depth is due to lenses in the form of white dwarfs - solid line - compared to the observation luminosity functions of the disk - top dotted line - and of the halo bottom dotted line.

\subsection{What if...?}

As it can be seen from Tables 2 and 3 our results are compatible with a halo where roughly $10 \%$ of the dark matter is in the form of white dwarfs. It is thus interesting to ask ourselves what would eventually be the observed luminosity function of halo white dwarfs if all the microlensing events (and the associated optical depth) are due to halo white dwarfs. To this regard we have conducted a series of simulations in which we have produced as many white dwarfs as needed in order to reproduce the optical depth found by the MACHO team, which roughly corresponds to $13 \pm 1$ microlenses. In all these simulations the standard IMF was adopted, since the biased mass functions AL and CSM1 studied in the previous sections produce roughly the same results with regard to the white dwarf luminosity function. It is worth noticing as well that the ad-hoc mass functions studied here overproduce white dwarfs either in the HDF or in the EROS survey in sharp contrast with the observations. The white dwarf luminosity function was then computed using the restrictions for the visual magnitude and proper motion detailed in Sect. 2.1. As was done in Sect. 3.1, forty independent realizations of this sample were computed to obtain the average white dwarf luminosity function along with its corresponding standard deviation for each luminosity bin. The result is shown in Fig. 9 as a solid line. For the sake of the comparison the observational disk and halo white dwarf luminosity functions of Bergeron et al. (1998) for the disk and of Torres et al. (1998) for the halo are also shown as dotted lines. As it can be seen in this figure, should white dwarfs be responsible of all the microlensing events the halo white dwarf luminosity function would be much closer to that of the disk rather than to that of the halo. It is, however, worth mentioning at this point that this is indeed an overestimate since for at least 4 lensing events it has been already shown that the lenses are not in the Galactic halo. In particular, one of the lenses resides in the galactic disk (Alcock et al. 2001a). Moreover, three of these are binary events (Afonso et al. 1988; Bennet et al. 1996; 
Alcock et al. 2001b) in the LMC or SMC. It is obvious, that it is not possible that all lensing events with lenses in the LMC and SMC are binary and, hence, it follows that a substantial fraction of single events must also be in the LMC or the SMC as first pointed out by Sahu (1994). However, it is true as well that this simulation reinforces the result that most of the microlensing events reported by the MACHO team are not halo white dwarfs and that another explanation must be found for these results.

\subsection{The role of the density profile}

To check the sensitivity of our results with respect to a different density profile we have done a final series of simulations in which the density profile of Navarro et al. (1997) was adopted. This density profile is characterized by the following expression

$\rho(r)=\frac{\rho_{\text {crit }} \delta_{\mathrm{c}}}{\left(r / r_{\mathrm{s}}\right)\left(1+r / r_{\mathrm{s}}\right)^{2}}$,

where $r_{\mathrm{s}}$ is the scale radius, $\delta_{\mathrm{c}}$ is an adimensional characteristic density, and $\rho_{\text {crit }}=3 H^{2} / 8 \pi G$ is the critical density of an Einstein-De Sitter universe. On the other hand, $r_{\mathrm{s}}$ is usually defined through the concentration parameter $c=R_{200} / r_{\mathrm{s}}$ as a function of the virial radius, $R_{200}$. In order to correctly reproduce the rotation curve of our Galaxy the following set of parameters is usually adopted: $c=10, R_{200}=180 \mathrm{kpc}$, which results in $r_{\mathrm{s}}=18 \mathrm{kpc}$. In this simulation, and as discussed previously, the adopted initial mass function was the standard one. We only computed the number of microlenses towards the LMC and not the results of the HDF or of the EROS experiment.

The different density profiles used here are displayed in Fig. 10. The solid line corresponds to the classical isothermal sphere density profile, the short dashed line to a power law with exponent $\gamma=2.0$, the long dashed line to a power law with $\gamma=3.4$ and the dashed-dotted line to the Navarro et al. (1997) density profile. The top panel shows the density profile as a function of the galactocentric $r$ coordinate, whereas the bottom panel shows the density profile projected towards the direction of the LMC. As it can be seen in the top panel of Fig. 10, all the density profiles, including that of Navarro et al. (1997), are much more concentrated towards the center of the Galaxy than the isothermal sphere. Additionally, the power law with $\gamma=3.4$ decreases much faster than the other three density profiles considered here. However, when projected towards the LMC, and for the distances of interest (those relevant for the study of the microlenses towards the LMC, obtained in the previous sections, $3 \mathrm{kpc}$ ), all four density profiles yield similar densities. Consequently, we do not expect large departures from what has been found in previous sections, and this is indeed the case. In particular, the results obtained using the Navarro et al. (1997) density profile are shown in Table 7, and it is evident from a cursory inspection that the results do not differ much from those shown in Table 3. Hence, the same conclusions apply, namely that the contribution of halo white dwarfs to the microlensing events towards the LMC must be small, independently of the adopted density profile.
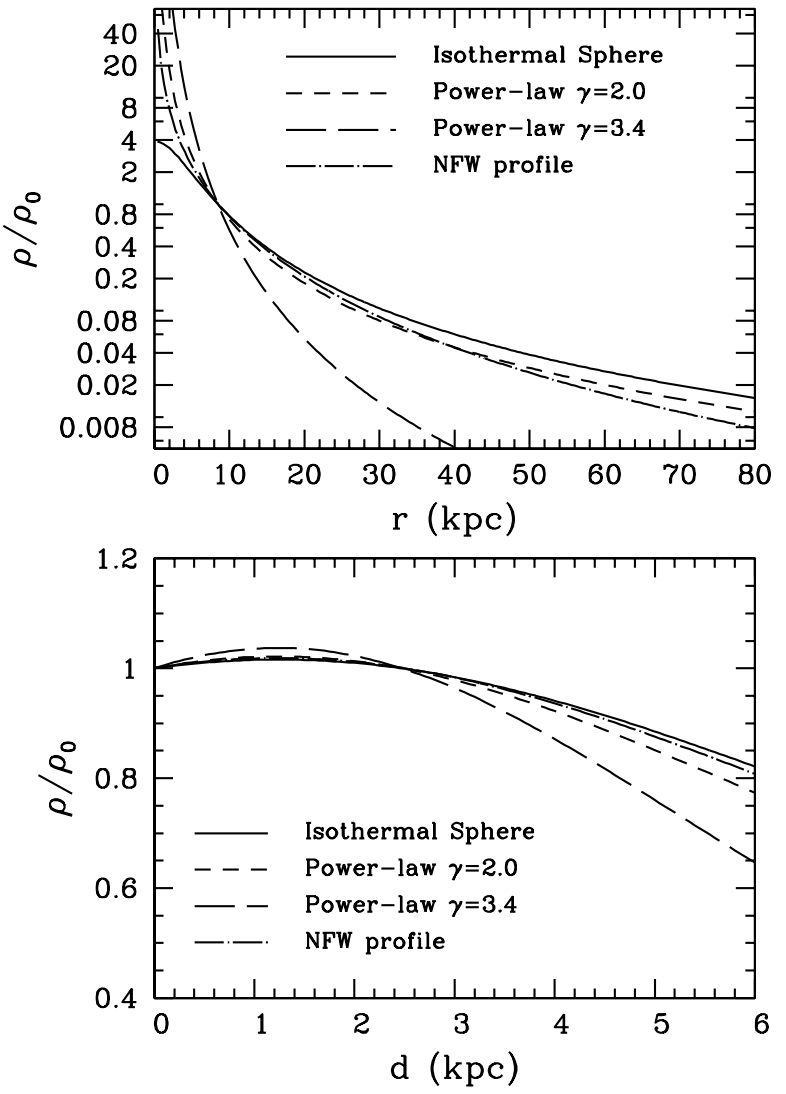

Fig. 10. The density profiles used in the simulations. The top panel shows the density profiles from the center of the Galaxy, whereas the bottom panel shows the density profiles towards the direction of the LMC.

\subsection{Miscellanea}

In order to check the robustness of our results we have conducted a series of numerical experiments in which, firstly, the distance to the LMC was varied by a $10 \%$ and, secondly, the tangential velocity of the resulting white dwarf population was also varied by a $10 \%$. In all the cases a standard IMF was used. The results are shown in Table 8. Our fiducial model is shown in Col. 3. The second column corresponds to a model in which a distance of $45 \mathrm{kpc}$ was adopted, whereas the model in which a distance of $55 \mathrm{kpc}$ was used is shown in Col. 4. Columns 5 and 6 correspond, respectively, to the models in which the tangential velocities were decreased $\left(V_{\tan }^{-}\right)$and increased $\left(V_{\tan }^{+}\right)$artificially by a $10 \%$. As can be seen there, the differences in all the cases are relatively small. For instance decreasing the distance to the LMC from 50 to 45 decreases the contribution of white dwarfs to the observed microlensing optical depth by $6 \%$, whereas the model in which the contribution of white dwarfs to the microlensing optical depth would be largest is that in which the tangential velocities of all the white dwarfs have been increased by $10 \%$. In this case the contribution amounts to a $13 \%$. Given all these considerations it seems unlikely that we could have underestimated the contribution of white dwarfs to the observed microlensing optical depth. 
Table 7. Summary of the results obtained for the microlensing events towards the LMC when the density profile of Navarro et al. (1997) is adopted. See text for more details.

\begin{tabular}{lccc}
\hline \hline & \multicolumn{3}{c}{ Standard } \\
\hline Magnitude & $17.5 \mathrm{mag}$ & $22.5 \mathrm{mag}$ & $27.5 \mathrm{mag}$ \\
\cline { 2 - 4 }$\left\langle N_{\mathrm{WD}}\right\rangle$ & $0 \pm 1$ & $0 \pm 1$ & $0 \pm 1$ \\
$\langle m\rangle\left(M / M_{\odot}\right)$ & 0.685 & 0.660 & 0.651 \\
$\langle\mu\rangle\left({ }^{\prime \prime} \mathrm{yr}^{-1}\right)$ & 0.158 & 0.062 & 0.019 \\
$\langle d\rangle(\mathrm{kpc})$ & 0.33 & 1.21 & 4.25 \\
$\left\langle V_{\mathrm{tan}}\right\rangle\left(\mathrm{km} \mathrm{s}^{-1}\right)$ & 248 & 360 & 386 \\
$\left\langle\hat{\mathrm{t}}_{\mathrm{E}}\right\rangle(\mathrm{d})$ & 18.5 & 23.2 & 40.5 \\
$\left\langle\tau / \tau_{0}\right\rangle$ & 0.064 & 0.061 & 0.060 \\
\hline
\end{tabular}

Table 8. Summary of the results obtained for a limiting magnitude of $m_{\mathrm{V}}=17.5^{\mathrm{mag}}$ and diferent values of the LMC distance and the tangential velocity.

\begin{tabular}{lccccc}
\hline \hline & $45 \mathrm{kpc}$ & $50 \mathrm{kpc}$ & $55 \mathrm{kpc}$ & $V_{\text {tan }}^{-}$ & $V_{\text {tan }}^{+}$ \\
\hline$\left\langle N_{\mathrm{WD}}\right\rangle$ & $0 \pm 1$ & $0 \pm 1$ & $0 \pm 1$ & $0 \pm 1$ & $0 \pm 1$ \\
$\langle\mu\rangle\left({ }^{\prime \prime} \mathrm{yr}^{-1}\right)$ & 0.030 & 0.016 & 0.017 & 0.010 & 0.041 \\
$\left\langle\tau / \tau_{0}\right\rangle$ & 0.063 & 0.101 & 0.086 & 0.092 & 0.132 \\
\hline
\end{tabular}

\section{Discussion and conclusions}

Using a Monte Carlo code we have computed self-consistently and simultaneously the theoretical expectations of the number of white dwarfs for four different observational results, namely the reported microlensing events towards the Large Magellanic Cloud (Alcock et al. 1997, 2000), the local halo white dwarf luminosity function (Torres et al. 1998), the results of the Hubble Deep Field (Ibata et al. 1999) and the results of the EROS experiment (Goldman et al. 2002). Our Monte Carlo simulator takes into account all the known observational biases and uses a thorough description of the properties of the Galactic halo. In our calculations we have simulated the halo white dwarf population for several halo ages (12, 14 and $16 \mathrm{Gyr})$. We have also used four different initial mass functions: a standard IMF (Scalo 1998), the two ad-hoc initial mass functions of Chabrier et al. (1996), and the biased initial mass function of Adams \& Laughlin (1996) - see Table 1.

We have found that none but the standard IMF is able to reproduce simultaneously the observations of the HDF, of the EROS experiment and of the local halo white dwarf luminosity function. In particular, the most extreme initial mass function of Chabrier et al. (1996) largely overproduces white dwarfs for both the EROS survey and for the HDF. More precisely, the EROS experiment in their survey did not find any candidate white dwarf whereas our simulations show that if this was the initial mass function of the halo they should have found around 12 white dwarfs. The same holds for the HDF, where a maximum of 4 white dwarfs should have been found, whereas our simulations using this IMF yield about 30 white dwarfs. Thus, this IMF is completely ruled out by observations. Regarding the more conservative IMF of Chabrier et al. (1996) our simulations predict $4 \pm 4$ white dwarfs for the HDF and $6 \pm 2$ for the EROS survey. Therefore, although this IMF is marginally compatible with the results of the HDF and with the local white dwarf luminosity function, it turns out that is incompatible with the results of the EROS survey. Since the Adams \& Laughlin (1996) initial mass function is very similar to the Chabrier et al. (1996) mass function the same conclusions apply to this IMF.

Regarding the microlensing events towards the LMC we have found that white dwarfs at most could be responsible for $\sim 1$ event for the case in which the standard IMF is adopted. This number increases up to $\sim 5$ events for the cases in which the "moderate" log-normal mass function of Chabrier et al. (1996) and of Adams \& Laughlin (1996) are used. The contribution of these events to the observed optical depth $\tau_{0}$ is, respectively, $\tau / \tau_{0} \sim 0.1,0.2$ and 0.3 . Hence, even if ad-hoc initial mass functions are adopted the total number of microlensing events produced by halo white dwarfs and the corresponding optical depth found by the MACHO team cannot be recovered when the observational biases and other constraints are taken into account. Moreover, the average duration of the simulated microlensing events for all the samples in which biased mass functions were used is considerably smaller than the observed one. In summary, the ad-hoc initial mass functions of Chabrier et al. (1996) and of Adams \& Laughlin (1996), which were tailored to fit the observations of the MACHO team, fail to reproduce the rest of the observational constraints and do not yield the required number of microlensing events (and, thus, do not significantly contribute to the optical depth). Consequently they should be disregarded until new (and unlikely) evidence would eventually appear.

We would like to emphasize at this point that all the above mentioned results are almost completely independent of the adopted halo age. Moreover, in order to check whether or not our results are sensitive to the density profile of the halo, we have extended our simulations using the density profile of Navarro et al. (1997). We have found that the microlensing results are not sensitive to the choice of the density profile because at the distances of interest all the density profiles studied here are coincident.

In summary, our results strongly suggest that a sizeable fraction of the dynamical mass in the halo of our Galaxy cannot be in the form of old hydrogen-rich white dwarfs. More specifically, for the case in which a standard initial mass function is adopted this fraction turns out to be of the order of $4 \%$ if we trust the results of the HDF or as low as $1 \%$ if the results of the EROS team are adopted. In any case the percentage obtained here is well below the claim of the MACHO team that $20 \%$ of the dark halo of the Galaxy is tied up in half-solar mass stellar bodies (Alcock et al. 2000), since the constraints set by red star counts (Flynn et al. 1996) discard the possibility of low-mass main sequence stars. On the other hand our results are in excellent agreement not only with the observations of the HDF and of the EROS experiment but also with the theoretical estimates of Graff et al. (1998), §10\%, Flynn et al. (2001), $2 \%$, and more recently Goldman et al. (2002), $\$ 4 \%$, and Zhao (2002), $\$ 4 \%$. Of course, the real nature of the reported microlensing events remains still a mystery.

Acknowledgements. Part of this work was supported by the MCYT grants AYA04094-C03-01 and 02, by the MCYT/DAAD grant HA2000-0038, and by the CIRIT. 


\section{References}

Abia, C., Domínguez, I., Straniero, O., et al. 2001, ApJ, 557, 126 Adams, F. C., \& Laughlin, G. 1996, ApJ, 468, 686

Afonso, C., Alard, C., Albert, J. N., et al. 1998, A\&A, 337, L17

Alcock, C., Allsman, R. A., Axelrod, T. S., et al. 1996, ApJ, 461, 84

Alcock, C., Allsman, R. A., Alves, D., et al. 1997, ApJ, 486, 697

Alcock, C., Allsman, R. A., Alves, D. R., et al. 2000, ApJ, 542, 281

Alcock, C., Allsman, R. A., Alves, D. R., et al. 2001, Nature, 414, 617

Alcock, C., Allsman, R. A., Alves, D. R., et al. 2001, ApJ, 552, 259

Bennett, D. P., Alcock, C., Allsman, R. A., et al. 1996, Nucl. Phys. Proc. Suppl., 51B, 152

Bergeron, P., Leggett, S. K., \& Ruiz, M. T. 1998, ApJ, 497, 294

Binney, J., \& Tremaine, H. 1987, Galactic Dynamics (Princeton: Princeton Univ. Press)

Canal, R., Isern, J., \& Ruiz-Lapuente, P. 1997, ApJ, 1997, 488, 35

Chabrier, G., Ségretain, L., \& Méra, D. 1996, ApJ, 468, L21

Dehnen, W., \& Binney, J. 1998, MNRAS, 298, 387

Díaz-Pinto, A., García-Berro, E., Hernanz, M., Isern, J., \& Mochkovitch, R. 1994, A\&A, 282, 86

Flynn, C., Gould, A., \& Bahcall, J. N. 1996, ApJ, 466, L55

Flynn, C., Sommer-Larsen, J., Fuchs, B., Graff, D. S., \& Salim, S. 2001, MNRAS, 322, 553

Flynn, C., Holopainen, J., \& Holmberg, J. 2003, MNRAS, 339, 817

García-Berro, E., Hernanz, M., Mochkovitch, R., \& Isern, J. 1988, A\&A, 193, 141

García-Berro, E., Torres, S., Isern, J., \& Burkert, A. 1999, MNRAS, 302,173

Gibson, B. K., \& Mould, J. R. 1997, ApJ, 482, 98

Goldman, B., Afonso, A., Alard, Ch., et al. 2002, A\&A, 389, 69

Gould, A., Flynn, C., \& Bahcall, J. N. 1998, ApJ, 499, 728

Graff, D. S., Laughlin, G., \& Freese, K. 1998, ApJ, 499, 7

Green, A. M., \& Jedamzik, K. 2002, A\&A, 395, 31

Gyuk, G., Dalal, N., \& Griest, K. 2000, ApJ, 535, 90

Hansen, B. M. S. 1999, ApJ, 520, 680

Hernanz, M., García-Berro, E., Isern, J., et al. 1994, ApJ, 434, 652

Ibata, R. A., Richer, H. B., Gilliland, R. L., \& Scott, D. 1999, ApJ, 524, L95

Iben, I., \& Laughlin, G. 1989, ApJ, 341, 312

Isern, J., García-Berro, E., Hernanz, M., Mochkovitch, R., \& Burkert, A. 1995a, in White Dwarfs, ed. D. Koester, \& K. Werner (Heidelberg: Springer Verlag), 19
Isern, J., García-Berro, E., Hernanz, M., Mochkovitch, R., \& Burkert, A. 1995b, in The Formation of the Milky Way, ed. E. J. Alfaro, \& A. J. Delgado (Cambridge: Cambridge University Press), 19

Isern, J., García-Berro, E., Hernanz, M., Mochkovitch, R., \& Torres, S. 1998, ApJ, 503, 239

Isern, J., García-Berro, E., Hernanz, M., \& Chabrier, G. 2000, ApJ, 528, 397

Knox, R. A., Hawkins, M. R. S., \& Hambly, N. C. 1999, MNRAS, 306,736

James, F. 1990, Comput. Phys. Commun., 60, 329

Lasserre, T., Afonso, C., Albert, J. N., et al. 2001, A\&A, 355, L39

Leggett, S. K., Ruiz, M. T., \& Bergeron, P. 1998, ApJ, 497, L294

Liebert, J., Dahn, C. C., \& Monet, D. G. 1988, ApJ, 332, 891

Majewski, S. R., \& Siegel, M. H. 2002, ApJ, 569, 432

Marković, D., \& Sommer-Larsen, J. 1997, MNRAS, 288, 733

Méndez, R. A., Minnitti, D., De Marchi, G., Baker, A., \& Couch, W. J. 1996, MNRAS, 283, 666

Navarro, J. F., Frenck, C. S., \& White, S. D. M. 1997, ApJ, 490, 493

Nelson, C. A., Cook, K. H., Axelrod, T. S., Mould, J. R., \& Alcock, C. 2002, ApJ, 573, 644

Noh, H.-R., \& Scalo, J. 1990, ApJ, 352, 605

Oppenheimer, B. R., Hambly, N. C., Digby, A. P., Hodgkin, S. T., \& Saumon, D. 2001, Science, 292, 698

Oswalt, T. D., Smith, J. A., Wood, M. A., \& Hintzen, P. 1996, Nature, 382, 692

Reylé, C., Robin, A. C., \& Créze, M. 2001, A\&A, L53

Sahu, K. 1994, Nature, 370, 275

Salati, P., Taillet, R., Aubourg, É., Palanque-Delabrouille, N., \& Spiro 1999, A\&A, 350, L57

Salaris, M., García-Berro, E., Hernanz, M., Isern, J., \& Saumon, D. 2000, ApJ, 544, 1036

Scalo, J. 1998, in The Stellar Initial Mass Function, ed. G. Gilmore, \& D. Howell (San Francisco), PASP Conf. Ser., 142, 201

Schmidt, M. 1968, ApJ, 151, 393

Torres, S., García-Berro, E., Burkert, A., \& Isern, J. 2002, MNRAS, 333, 971

Torres, S., García-Berro, E., \& Isern, J. 1998, ApJ, 508, L71

Winget, D. E., Hansen, C. J., Liebert, J. G., et al. 1987, ApJ, 315, L77

Wu, X. 1994, ApJ, 435, 66

Zhao, H. 2002, MNRAS, 336, 159 